\title{
Image processing applications through a variational perceptually-based color correction related to Retinex
}

\author{
Javier Vazquez-Corral' ${ }^{\text {, Syed Waqas Zamir }}{ }^{1}$, Adrian Galdran ${ }^{2}$, David Pardo ${ }^{3,4,5}$, Marcelo Bertalmío ${ }^{1}$; \\ ${ }^{1}$ Information and Communication Technologies Department, Universitat Pompeu Fabra, Barcelona, Spain \\ ${ }^{2}$ Tecnalia, Computer Vision Group, Derio, Spain \\ ${ }^{3}$ Department of Applied Mathematics, Statistics, and Operational Research, University of the Basque Country, Leioa, Spain. \\ ${ }^{4}$ BCAM, Basque Center for Applied Mathematics, Bilbao, Spain \\ ${ }^{5}$ IKERBASQUE, Basque Foundation for Science, Bilbao, Spain
}

\begin{abstract}
The retinex theory of color vision has been a light of inspiration for color researchers since its inception 50 years ago. It has been adapted to work for different goals such as shadow removal, high dynamic range imaging, or computational color constancy. Back in 2007 a variational perceptually-based color correction model related to Retinex was presented by Bertalmio and colleagues. In this paper we first comment on this model and later we review different image processing applications that have been obtained by performing small modifications to it, namely color gamut mapping and image dehazing.
\end{abstract}

\section{Introduction}

The Retinex theory of color vision [9] has been a very powerful influence for color researchers for almost 50 years. Retinex postulate can be over-summarised to the following idea: the colour of an object is not determined by the spectral composition of the light reaching to the eye, but is determined by the comparison between the three lightness values generated by this spectra at each cone photoreceptor and the values generated by a white stimulus spectra (considered to be the spectra of maximum radiance) in all three bands. Edwin Land hypothesized that this effect was happening either at the retina, the cortex, or at both levels, and therefore he created the portmanteau word 'Retinex' to express the effect.

Therefore, the main question that arises is: how do humans compare a particular color to a white reference that might be far away in the scene? Land and McCann [10] solved this issue by comparing points of the scene that are far apart through paths. This path computation allowed the authors to define three main mechanisms that should be accomplished: ratio, product, and reset. The product and ratio mechanisms are based on their proposal for the computation of the aforementioned comparison. First, this comparison is considered to be the ratio between the points, and second, this ratio is approximated through local comparisons among the path, i.e. the ratio of the two end points can be decomposed as the sequential product of ratios of each point of the path to the following one. This leads to the reset postulate that claims that the ratio between any point and its sequential one should be smaller than a unity. If this does not happen, it means that the radiance of the latter point is larger than the radiance of the previous point, and we should reset the sequential product of ratios to one. In this way, for any path, we will be always computing the ratio between the end point and the point with maximum value along the path, which is the approximation to the maximum radiance point [12].

Retinex has been characterized using diverse mathematical formulations during all these years. The straightforward random path-based computation presented by Land and McCann [10] has been modified to consider various models such as random sprays [13], Brownian motion [11,14], or swarn termites movement [15]. Retinex has been also mathematically defined using minimizations based on the Laplacian of the image [6] or variational-based energy minimizations [7].

Bertalmío, Caselles, Provenzi and Rizzi presented a variational perceptually-based color correction model [2] that was later proved to exhibit the main mechanisms of the original Retinex explained above: ratio, product, and reset [1]. In this paper, we will review on this particular method and its modifications to deal with different image processing applications. In particular, this model have been adapted to deal with color gamut mapping and image dehazing.

This paper is organized as follows. In the next section we will review the main characteristics of Bertalmío -et al.- model. Later in sections 3 and 4 , we will explain the modifications performed to the model to deal with color gamut mapping and image dehazing, respectively. Finally, conclusions are summed up in the last section.

\section{Variational perceptually-based color correc- tion}

Bertalmío and colleagues [2] proposed an image energy functional whose minimization complies with some very basic visual perception principles, namely those of grey-world, locality and not excessive departure from the original data:

$$
\begin{aligned}
E(I)=\frac{\alpha}{2} \sum_{x}\left(I(x)-\frac{1}{2}\right)^{2}- & \frac{\gamma}{2} \sum_{x} \sum_{y} w(x, y)|I(x)-I(y)| \\
& +\frac{\beta}{2} \sum_{x}\left(I(x)-I_{0}(x)\right)^{2},
\end{aligned}
$$

where $\alpha, \beta$ and $\gamma$ are constant and positive weights, $I$ is a color channel $(R, G$ or $B), w(x, y)$ is a normalized Gaussian kernel of standard deviation $\sigma$, and $I(x)$ and $I(y)$ are two intensity levels at pixel locations $x$ and $y$ respectively.

This model can be understood as a competition between three different terms. With a positive sign, there are two attachments: one to the original image and another to $\frac{1}{2}$, which is the expected 
mean of the image after white patch. With a negative sign, there is a contrast maximization term. In this way, the images resulting from the model represent a contrast-enhanced version of the original image without deviating too far it and staying closer to $\frac{1}{2}$.

The authors proved that the solution to the minimization of Eq. (1) can be found as the steady state of the evolution equation

$$
I^{k+1}(x)=\frac{I^{k}(x)+\Delta t\left(\frac{\alpha}{2}+\beta I_{0}(x)+\frac{\gamma}{2} R_{I^{k}}(x)\right)}{1+\Delta t(\alpha+\beta)},
$$

where the initial condition is $I^{k=0}(x)=I_{0}(x)$. The function $R_{I^{k}}(x)$ indicates the contrast function:

$$
R_{I^{k}}(x)=\frac{\sum_{y \in \mathfrak{I}} w(x, y) s\left(I^{k}(x)-I^{k}(y)\right)}{\sum_{y \in \mathfrak{I}} w(x, y)}
$$

where $x$ is a fixed image pixel and $y$ varies across the image. The slope function $s()$ is a regularized approximation to the sign function, which appears as it is the derivative of the absolute value function in the second term of the functional; in the original paper the authors choose for $s()$ a polynomial of degree 7. In this work we review two image processing applications where the model of Bertalmío -et al.- has been adapted to work, namely color gamut mapping and image dehazing.

\section{Color gamut mapping}

Color gamut mapping deals with the problem of modifying the gamut of an input image to make it consistent with the gamut of a particular device (printer, screen, projector, etc...). Generally, two different possibilities are considered: i) the gamut of the image should be reduced to fit into a smaller destination gamut (called gamut reduction), or ii) the gamut of the image should be increased to better cover a bigger destination gamut (referred as gamut extension). In a recent work, Zamir -et al.- [18] modified the model of [2] to perform gamut reduction and extension; this involved two significant modifications. First, they considered the mean of the original image as the expected mean for the final image (this modification was already applied in [3]). In this way, the new functional was defined as

$$
\begin{array}{r}
E(I)=\frac{\alpha}{2} \sum_{x}(I(x)-\mu)^{2}-\frac{\gamma}{2} \sum_{x} \sum_{y} w(x, y)|I(x)-I(y)| \\
+\frac{\beta}{2} \sum_{x}\left(I(x)-I_{0}(x)\right)^{2},
\end{array}
$$

where $\mu$ represents the mean of the original image. This implies, that the evolution equation for Eq. 4 can be written as

$$
I^{k+1}(x)=\frac{I^{k}(x)+\Delta t\left(\mu \alpha+\beta I_{0}(x)+\frac{\gamma}{2} R_{I^{k}}(x)\right)}{1+\Delta t(\alpha+\beta)}
$$

Second, the authors showed that the sign of the contrast term modifies the behavior of the model, so that it performs gamut reduction when the sign is positive and gamut extension when it is negative. Let us explain in detail how they used this observation in both cases.

\section{Gamut reduction}

Authors devised a double-iterative process for the gamut reduction case in order to select the best weight to be applied to the contrast term. The double iterative process works as follows. At each iteration, Eq. (5) is run for some particular $\alpha, \beta$, and $\gamma$ until the steady state is reached. The steady state of each iteration provides some pixels of the final result. At iteration 1, values are set to $\beta=1, \alpha=0$, and $\gamma=0$, and therefore the original image is obtained as the steady state. At this point, those pixels already belonging to the destination gamut are selected for the final image and leaved untouched for the following iterations. At iteration $2, \gamma$ is decreased (for example, $\gamma=-0.05$ ) and $\alpha$ is increased in relation to $\gamma$ by $\frac{|\gamma|}{20}$. Eq. (5) is run again until steady state, and it is checked whether any of the colors that were outside the gamut at the previous iteration have been moved inside the destination gamut. If this is the case, those pixels are selected for the final image and leaved unmodified for the following iterations. This procedure continues by decreasing $\gamma$ (and increasing $\alpha$ accordingly) until all the out-of-gamut colors come inside the destination gamut.

\section{Gamut extension}

In this case, the authors proposed to extend the gamut of the original image by using a unique gamma value. For selecting the gamma value, Eq. (5) is applied to different values of $\gamma$ and let the original gamut exceed the destination gamut upto a certain threshold level $\left(T_{e}\right)$. Later on, the colors that were placed outside the destination gamut in the previous stage are mapped back inside using the gamut reduction algorithm presented previously.

An example of both gamut reduction and extension with the method of Zamir -et al.- is shown in Figure 1. The top row shows an original image (left) and its gamut reduced version (right), while the bottom row presents an original image (left) and its gamut extended version (right).

\section{Joint gamut reduction and gamut extension}

Although much research has been devoted independently to gamut reduction and gamut extension, the more general gamut mapping case where the intersection of source and target gamut is not equal to one of the two gamuts has received little attention. To fill this gap, Vazquez-Corral and Bertalmío [17] recently showed that by defining a smooth transition on the contrast enhancement parameter over the color space, it is possible to simultaneously reduce the input gamut in some areas of the color space while increasing it in others without introducing color artifacts or halos. This ability to modify at will the image colors allowed them to convert the palette of colors of an original image into the palette of colors of an unrelated second image, as can be seen in Figure 2. This figure contains the original image (left), the target image (right), and the result obtained by Vazquez-Corral and Bertalmío [17] (center).

\section{Image dehazing}

Image dehazing, i.e. robustly recovering visual information in bad weather conditions, is currently a key pre-processing task for many different computer vision applications such as tracking, video surveillance, or autonomous driving cars. Many different methods have been defined over the last years to handle this problem, with the majority employing some form of constrast or saturation enhancement [16], [5]. This is the key connection between the model of [2] and image dehazing methods.

Galdran -et al.- [4] modified the model of [2] in two different 

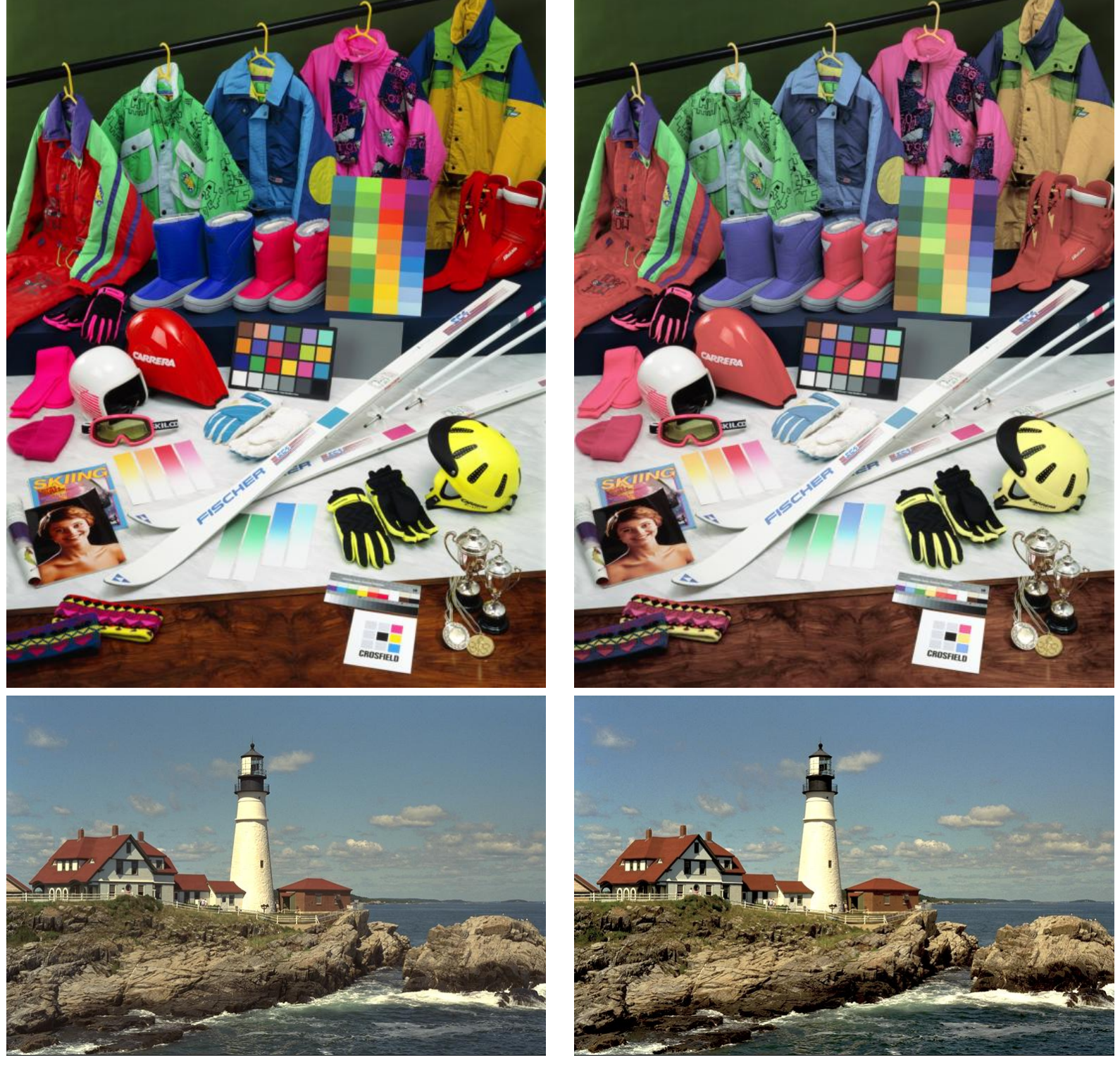

Figure 1: Top row: Original image (left) and gamut reduced version (right) by Zamir -et al.- method [18]. Bottom row: Original image (left) and gamut extended version (right) by Zamir -et al.- method [18].
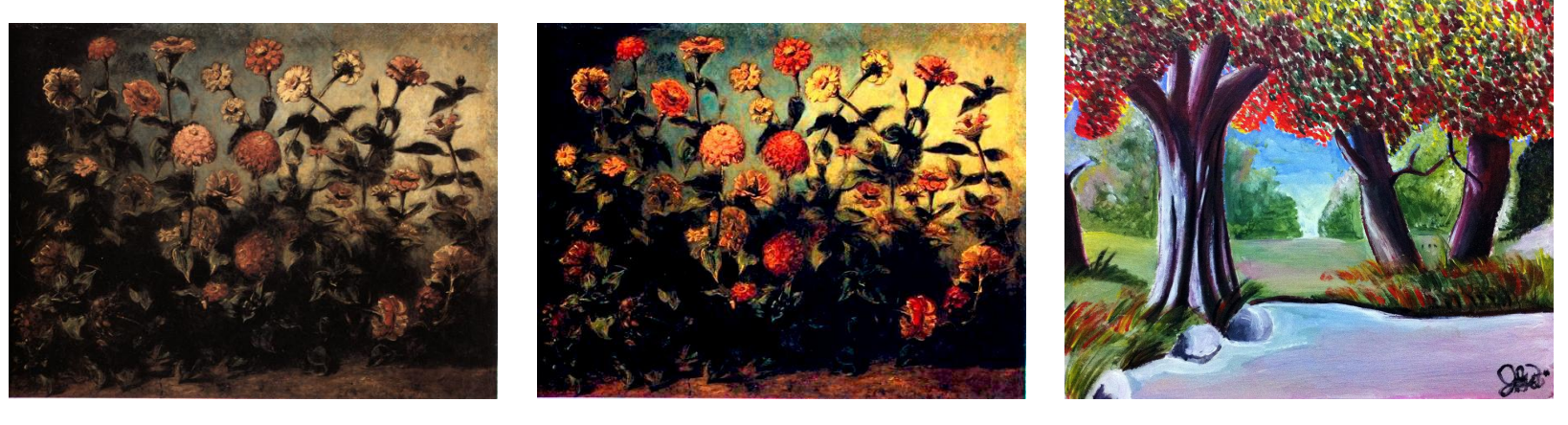

Figure 2: Original image (left), target image (right), and the result of the color modification performed by [17](center). 
manners in order to perform image dehazing. First, they modified the expected value of the image mean. To this end, they applied the Koschsmeider law of image formation in order to obtain the expected mean of the clean image instead of the expected mean of the original image. Mathematically, they started considering the Koschsmieder law [8], that is written channel-wise as

$$
\mathrm{I}^{j}(x)=\mathbf{J}^{j}(x) \mathrm{t}(x)+(1-\mathrm{t}(x)) A^{j},
$$

where $j \in\{R, G, B\}, x$ is a pixel location, $\mathrm{I}(x)$ is the observed intensity, $\mathrm{J}(x)$ is the scene radiance, corresponding to the clean image, transmission $\mathrm{t}(x)$ is a scalar quantity that is inversely related to the scene's depth,and A is the airlight.

Galdran -et al.- rearranged the previous equation and took the average of each term leading to

$$
\operatorname{mean}\left(\mathrm{J}^{j} \cdot \mathrm{t}\right)=\operatorname{mean}\left(\mathrm{I}^{j}\right)-\operatorname{mean}\left((1-\mathrm{t}) A^{j}\right) .
$$

From this point, two crutial assumptions are considered: i) On a haze-free image, we can expect colors to be independent of where the object is located in the scene. Thus, we can assume that $\mathrm{J}^{j}$ and $t$ are uncorrelated, and ii) $t$ has a uniform distribution across the image, i.e., depth values are equally distributed. From these two assumptions, mean $\left(\mathrm{J}^{j}\right)$ can be estimated as:

$$
\operatorname{mean}\left(\mathrm{J}^{j}\right) / 2 \approx \operatorname{mean}\left(\mathrm{I}^{j}\right)-(1 / 2) \operatorname{mean}\left(\mathrm{A}^{j}\right) .
$$

Finally, as the airlight A can be roughly approximated by the maximum intensity value on each component, the estimation of the mean of the clean image is:

$$
\mu^{j}=\operatorname{mean}\left(\mathrm{J}^{j}\right) \approx 2 \operatorname{mean}\left(\mathrm{I}^{j}\right)-\mathrm{A}^{j} .
$$

Second, they introduced two new negative competing terms to couple the color channels. They correspond to the two interchannel contrast differences (for example, in case of the red channel, red versus green and red versus blue). Mathematically, they define the inter-channel contrast as

$$
\frac{\eta}{2} \int_{x, y} \omega(x, y)\left|\mathrm{I}^{j}(x)-\mathrm{I}^{j+1}(y)\right|
$$

where as in the original model od Bertalmío -et al.- [2] $w(x, y)$ is a normalized Gaussian kernel of standard deviation $\sigma, \eta$ is a constant and positive weight, and $I^{j}(x)$ and $I^{j+1}(y)$ are two intensity levels at pixel locations $x$ and $y$ from the color channels $j$ and $j+1$, respectively. Galdran and colleagues showed that by maximizing these new two terms (via a minimization of the energy functional) they increase the difference in values between the channels, therefore increasing the saturation of the images, which is related to the dark channel image dehazing idea [5].

The ensemble of both modifications lead to the following image energy functional

$$
\begin{aligned}
& E\left(\mathrm{I}^{j}\right)=\frac{\alpha}{2} \int_{x}\left(\mathrm{I}^{j}(x)-\mu^{j}\right)^{2}+\frac{\beta}{2} \int_{x}\left(\mathrm{I}^{j}(x)-\mathrm{I}_{0}^{j}(x)\right)^{2} \\
& -\frac{\gamma}{2} \int_{x, y} \omega(x, y)\left|\mathrm{I}^{j}(x)-\mathrm{I}^{j}(y)\right| \\
& -\frac{\eta}{2} \int_{x, y} \omega(x, y)\left|\mathrm{I}^{j}(x)-\mathrm{I}^{j+1}(y)\right| \\
& -\frac{\eta}{2} \int_{x, y} \omega(x, y)\left|\mathrm{I}^{j}(x)-\mathrm{I}^{j+2}(y)\right|,
\end{aligned}
$$

where $j, j+1$, and $j+2$ represent the three color channels.

This new energy functional has as solution the steady state of the following equation

$$
\begin{aligned}
& I^{k+1}(x)=\frac{I^{k}(x)}{1+\Delta t(\alpha+\beta)} \\
& +\frac{\Delta t\left(\frac{\alpha}{2}+\beta I_{0}(x)+\frac{\gamma}{2} R_{I^{j}, I^{j}}(x)\right)}{1+\Delta t(\alpha+\beta)} \\
& +\frac{\Delta t\left(\frac{\eta}{2}\left(R_{I^{j}, I^{j+1}}(x)+R_{I^{j}, I^{j+2}}(x)\right)\right)}{1+\Delta t(\alpha+\beta)}
\end{aligned}
$$

In this case $R\left(\mathrm{I}^{j}, \mathrm{I}^{j+1}\right)$ is defined as

$$
R_{I^{j}, I^{j+1}}(x)=\frac{\sum_{y \in \mathfrak{I}} w(x, y) s\left(I^{j}(x)-I^{j+1}(y)\right)}{\sum_{y \in \mathfrak{I}} w(x, y)}
$$

Figure 3 illustrates the image enhancement produced by the method devised in [4] by Galdran et. al. (right images) over the original hazy images showing the cities of Bilbao and London (left images).

\section{Conclusions and further work}

In this paper we have reviewed the variational perceptuallybased color correction model of Bertalmío and colleagues [2], and its applications to image processing applications, such as color gamut mapping and image dehazing. Further work is being performed to adapt the energy functional of [2] to take into account different priors such as natural image statistics or more complex color constancy formulations, and to improve its computational time.

\section{Acknowledgements}

J. Vazquez-Corral. S.W. Zamir, and M. Bertalmío were supported by European Research Council, Starting Grant ref. 306337, by the Spanish government, grant ref. TIN2012-38112, and by the Icrea Academia Award. D. Pardo was partially funded by the Project of the Spanish Ministry of Economy and Competitiveness with reference MTM2013-40824-P, the BCAM "Severo Ochoa" accreditation of excellence SEV-2013-0323, the CYTED 2011 project 712RT0449, and the Basque Government Consolidated Research Group Grant IT649-13 on "Mathematical Modeling, Simulation, and Industrial Applications (M2SI)"

\section{References}

[1] Marcelo Bertalmío, Vicent Caselles, and Edoardo Provenzi. Issues about retinex theory and contrast enhancement. International Journal of Computer Vision, 83(1):101-119, 2009.

[2] Marcelo Bertalmío, Vicent Caselles, Edoardo Provenzi, and Alessandro Rizzi. Perceptual color correction through variational techniques. IEEE Transactions on Image Processing, 16(4):1058-1072, 2007.

[3] Sira Ferradans, Marcelo Bertalmío, Edoardo Provenzi, and Vicent Caselles. An analysis of visual adaptation and contrast perception for tone mapping. IEEE Trans. Pattern Anal. Mach. Intell., 33(10):20022012, 2011.

[4] Adrian Galdran, Javier Vazquez-Corral, David Pardo, and Marcelo Bertalmío. Enhanced variational image dehazing. SIAM J. Imaging Sciences, 8(3):1519-1546, 2015. 

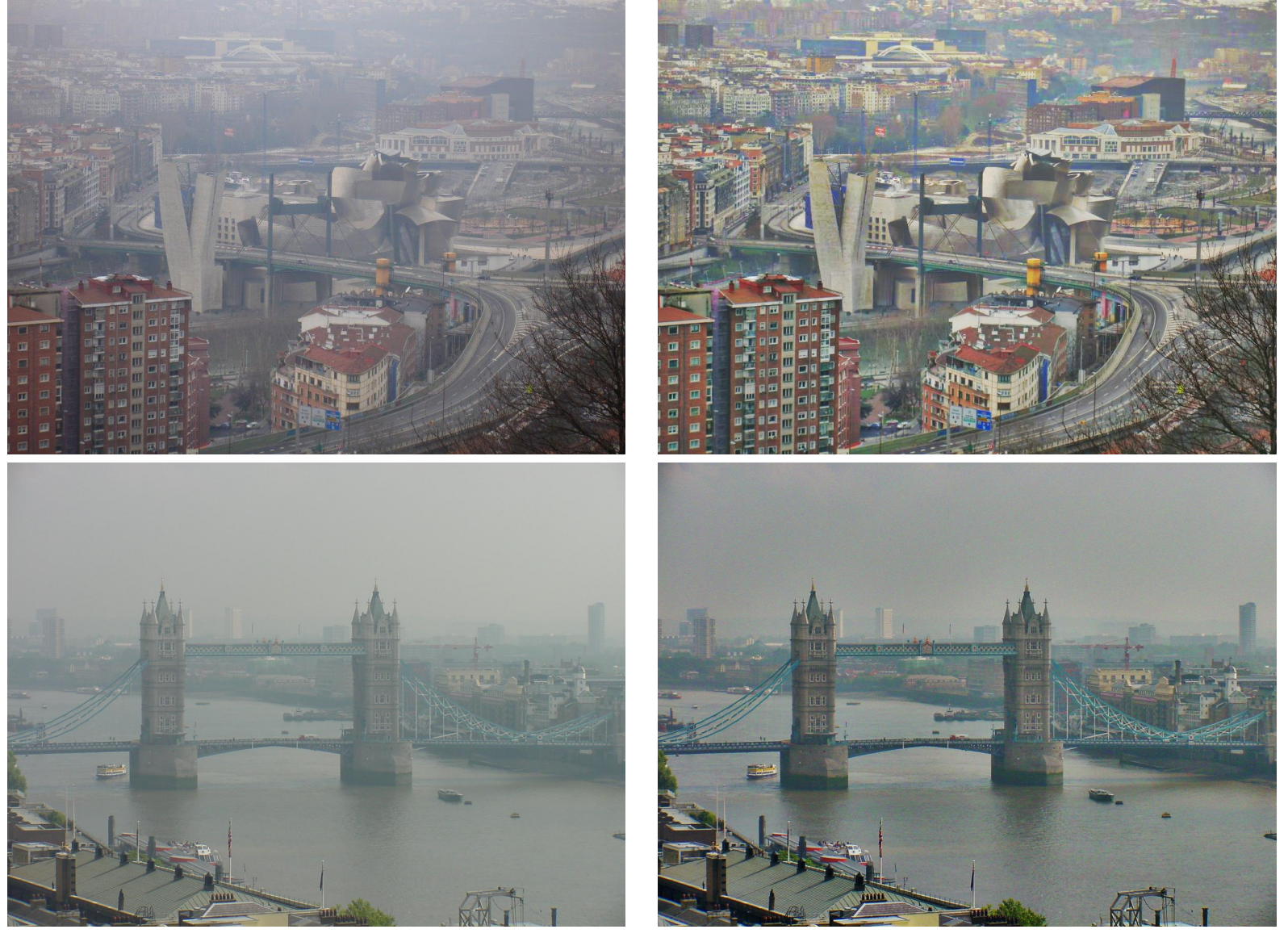

Figure 3: Original images (left) and images dehazed using Galdran -et al.- method [4]. 
[5] Kaiming He, Jian Sun, and Xiaoou Tang. Single image haze removal using dark channel prior. IEEE Trans. Pattern Anal. Mach. Intell., 33(12):2341-2353, 2011.

[6] Berthold K.P. Horn. Determining lightness from an image. Computer Graphics and Image Processing, 3(4):277 - 299, 1974.

[7] Ron Kimmel, Michael Elad, Doron Shaked, Renato Keshet, and Irwin Sobel. A variational framework for retinex. International Journal of Computer Vision, 52(1):7-23, 2003.

[8] Harald Koschmieder. Theorie der horizontalen Sichtweite: Kontrast und Sichtweite. Keim \& Nemnich, 1925.

[9] Edwin H. Land. The retinex. American Scientist, 52(2):247-264, 1964.

[10] Edwin H. Land and John J. McCann. Lightness and retinex theory. Journal of the Optical Society of America, pages 1-11, 1971.

[11] Roberto Montagna and Graham D. Finlayson. Constrained pseudobrownian motion and its application to image enhancement. J. Opt. Soc. Am. A, 28(8):1677-1688, Aug 2011.

[12] Edoardo Provenzi, Luca De Carli, Alessandro Rizzi, and Daniele Marini. Mathematical definition and analysis of the retinex algorithm. J. Opt. Soc. Am. A, 22(12):2613-2621, Dec 2005.

[13] Edoardo Provenzi, Massimo Fierro, Alessandro Rizzi, Luca De Carli, Davide Gadia, and Daniele Marini. Random spray retinex: A new retinex implementation to investigate the local properties of the model. Image Processing, IEEE Transactions on, 16(1):162-171, Jan 2007.

[14] Alessandro Rizzi, Daniele Marini, Luigi Rovati, and Franco Docchio. Unsupervised corrections of unknown chromatic dominants using a brownian-path-based retinex algorithm. Journal of Electronic Imaging, 12(3):431-441, 2003.

[15] Gabriele Simone, Giuseppe Audino, Ivar Farup, Fritz Albregtsen, and Alessandro Rizzi. Termite retinex: a new implementation based on a colony of intelligent agents. Journal of Electronic Imaging, 23(1):013006, 2014.

[16] Robby T. Tan. Visibility in bad weather from a single image. In 2008 IEEE Computer Society Conference on Computer Vision and Pattern Recognition (CVPR 2008), 24-26 June 2008, Anchorage, Alaska, USA, 2008.

[17] Javier Vazquez-Corral and Marcelo Bertalmío. Perceptually-inspired gamut mapping between any gamuts with any intersection. In AIC Midterm meeting, 2015.

[18] Syed Waqas Zamir, Javier Vazquez-Corral, and Marcelo Bertalmío. Gamut mapping in cinematography through perceptually-based contrast modification. J. Sel. Topics Signal Processing, 8(3):490-503, 2014.

\section{Author Biography}

Javier Vazquez-Corral received the Ph.D. degree in computer science from the Universitat Autònoma de Barcelona (UAB), Bellaterra, Spain, in 2011. He is a Postdoctoral fellow at Universitat Pompeu Fabra $(U P F)$ in Barcelona. His research interests are related to the use of color in image processing and computer vision problems. He is also interested in bridging the gap between color in the human brain and its use in computer-vision applications.

Syed Waqas Zamir received his B.Sc. in computer engineering from COMSATS Institute of Information Technology, Pakistan, in 2010. It was later complemented by an M.Sc. in digital signal processing from Queen Mary, University of London, UK, in 2011. Currently, he is a Ph.D.student in image processing at the Universitat Pompeu Fabra,
Barcelona, Spain. His research interests include image processing, color vision and computer vision. In particular, he is working in the development of perceptually inspired gamut mapping algorithms.

Adrian Galdran received a B.S. degree in Mathematics from the University of Valencia, Spain, in 2008, and a M.S. Mathematics Investigation from the University of Valencia and the Polytechnical University of Valencia. On 2011, he got an M.S. in Mathematical Modelization, Statistics and Computation at the Basque Country University. He is now finishing a PhD on Mathematical Image Processing, at the research company Tecnalia Research \& Innovation, in collaboration with the Department of Applied Mathematics at the University of the Basque Country. He is interested on image preprocessing, specially under adverse conditions, and variational techniques for computer vision and image processing applications.

David Pardo is a Research Professor at Ikerbasque, the University of the Basque Country UPV/EHU, and the Basque Center for Applied Mathematics (BCAM). He has published over 110 research articles and he has given over 190 presentations. In 2011, he was awarded as the best Spanish young researcher in Applied Mathematics by the Spanish Society of Applied Mathematics (SEMA). He leads a European Project on subsurface visualization, several national research projects, as well as research contracts with national and international companies. He is now the PI of the research group on Mathematical Modeling, Simulation, and Industrial Applications (M2SI). His research interests include computational electromagnetics, petroleum-engineering applications (borehole simulations), adaptive finite-element and discontinuous Petrov-Galerkin methods, multigrid solvers, image restoration algorithms, and multiphysics and inverse problems.

Marcelo Bertalmío received the Ph.D. degree in electrical and computer engineering from the University of Minnesota in 2001. He is an Associate Professor at University Pompeu Fabra in Barcelona, Spain. His interests are Image Processing and Computer Vision for digital cinema applications, although he prefers the (analog) films of Joseph L. Mankiewicz and Ernest Lubitsch. 